\title{
The Post-Transcriptional mRNA Editing Analysis of cox3 Mitochondrial Gene in Fern Asplenium nidus Reveals Important Features
}

\author{
Sebastian Panarese, Guglielmo Rainaldi \\ Department of Biochemistry and Molecular Biology, University of BARI “Aldo Moro”, Bari, Italy. \\ Email: g.rainaldi@biologia.uniba.it
}

Received July 18 ${ }^{\text {th }}$, 2011; revised August 20 ${ }^{\text {th }}, 2011$; accepted September $5^{\text {th }}, 2011$.

\begin{abstract}
In the mitochondria and chloroplasts of flowering plants (angiosperms), transcripts of protein-coding genes are altered after synthesis so that their final primary nucleotide sequence differs from that of the corresponding DNA sequence. This posttranscriptional mRNA editing consists almost exclusively of $C$-to- $U$ substitutions (direct) and less frequently of $U$-to-C substitution (reverse). Editing occurs predominantly within coding regions, mostly at isolated $C$ residues, and usually at first or second positions of codons, thereby almost always changing the amino acid from that specified by the unedited codon. Editing may also create initiation and termination codons. The effect of C-to-U RNA editing in plants is to make proteins encoded by plant organelles more similar in sequence to their non plant homologs, then specific C-to$U$ editing events are essential for the production of functional plant mitochondrial proteins. Our attention has been devoted to the study of the mRNA editing in cox3 mitochondrial gene of fern Asplenium nidus. This study reveals the extreme importance of both $C$-to- $U$ and $U$-to-C substitutions for protein expression.
\end{abstract}

Keywords: mtDNA, Plant, Monilophytes, Asplenium nidus

\section{Introduction}

Mitochondrial genomes of land plants have been fully sequenced and characterized in several species belonging to the Briophytes (Marchantia polymorpha [1] and Physcomitrella patens [2]) and Spermatophytes (Arabidopsis thaliana [3], Beta vulgaris [4], Oryza sativa [5], Brassica napus [6], Zea mays [7], Nicotiana tabacum [8] and Triticum aestivum [9]).

The comparison of organization, structure and expression between Spermatophyte mitochondrial genomes reveals several homogeneous features which can be summarized as follows: i) the presence of repeated sequences, ii) a heterogeneous structure, iii) the presence of DNA segments of extra mitochondrial origin (mainly chloroplastic) carrying in some cases active genes (usually for tRNAs) [10-12], iv) the editing of transcription products of structural genes, v) an incomplete set of tRNA genes.

To gain more knowledge on the mitochondrial biogenesis of Monilophytes, in particular respect to editing process, we chose plants of a filicales family, the fern Asplenium nidus, available at the Botanical Garden of the University of Bari.
In our previous investigations [13] we verified high level of editing process in filicales with to respect Spermatophytes plants. In our system, the editing analysis of cox3 mitochondrial gene confirms this hypothesis.

\section{Materials and Methods}

\subsection{Sources of Mitochondrial DNA}

Two alternative procedures for the isolation of organelles have been developed depending on the tissue used as starting material: roots or leaves. In the former the soil contained in the thick network of roots was removed by hand and washed in distilled water. This step was followed by drying the roots on filter paper and weighing and wrapping them with a double layer of sterile gauze. After washing several times with sterile water, the roots were suspended in sterile buffer (Mannitol 0,4 M, Mops 25 mM, EGTA 1 mM, PVP 1\% pH 7,8) and homogenized in a blender with five hits every five seconds at medium speed.

Fractionation, lysis of organelles and DNA extraction were as reported by Hanson [14].

The isolation of organelles and DNA from green 
leaves (leaf-procedure) was carried out using the same protocol with few modifications [14].

The mitochondrial DNA isolated by the two alternative procedures showed significant different levels of chloroplast contamination, as judged from the PCR amplification of highly conserved chloroplast regions [15].

Using as a template the mtDNA prepared from the roots, where the copy number of plastid DNA are reduced [16], no amplified products could be detected. The results of these experiments are reported in Figure 1.

The leaf procedure was used mainly for the isolation of total RNA employed for reverse transcription and cDNA synthesis and for the investigation concerning editing of cox3 gene transcripts.

\subsection{Total RNA Extraction from A. Nidus}

RNeasy Plant Mini Kit (Qiagen) was used for the purification of total RNA from fresh or frozen plant tissues. An accurate photometric detection of starting material was essential in order to obtain optimal RNA yield and purity. A maximum amount of $100 \mathrm{mg}$ plant material was generally processed.

\subsection{RT-PCR}

RT-PCR experiments were carried out in a single step by means of SuperScript One-Step RT-PCR with Platinum Taq. Components for both cDNA synthesis and PCR

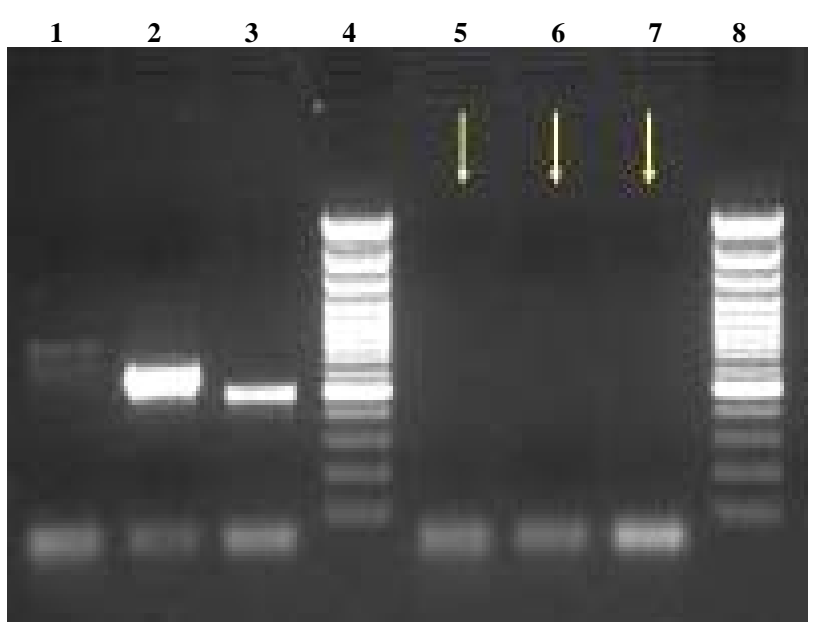

Figure 1. Multiple test of amplification performed on nucleic acids extracted with the two different procedures. The panels show two groups of three amplifications carried out with the same pairs of universal primers (BA48557-A49291, BA49317-A49855, BA49873-A50272, [14] for the detection of cpDNA in the nucleic acid fractions isolated by the two procedures. Lanes 1-3: template isolated by the leaf procedure. 5-7: template (arrows) isolated by the root procedure. 4 and 8: 1 Kb DNA ladder (3000, 2000, 1500, 1200, 1031, 900, 800, 700, 600, 500, 400, 300, 200, 100 bp). were combined in a single tube, using gene-specific primers and target RNAs from total RNA. Reverse transcription automatically followed PCR cycling without additional steps. The system consisted of two major components: RT/Platinum Taq Mix and 2X Reaction Mix where the RT/Platinum Taq Mix contained a mixture of SuperScript II Reverse Transcriptase and Platinum Taq DNA Polymerase for optimal cDNA synthesis and PCR amplification respectively.

The following cycling conditions were established: cDNA synthesis pre-denaturation (1X) at $50^{\circ} \mathrm{C}$ for $15-30$ '; $94^{\circ} \mathrm{C}$ for 2'; PCR amplification (40X) at $94^{\circ} \mathrm{C}$ for $20^{\prime \prime}$; $50^{\circ} \mathrm{C}-60^{\circ} \mathrm{C}$ for $30^{\prime \prime} ; 68^{\circ} \mathrm{C}-72^{\circ} \mathrm{C}$ for $1^{\prime} / \mathrm{kb}$; final extension $(1 \mathrm{X})$ at $72^{\circ} \mathrm{C}$ for $7^{\prime}$.

\subsection{DNA Cloning in Ta Vectors}

The cloning of dsDNA fragments was carried out using pGEM-T Easy Vector Systems and following the manufacturer instructions.

\subsection{DNA Sequencing and Determination of Editing Rates}

Sequencing of individual cDNA of 23 clones was performed automatically with the Big Dye1 Terminator Cycle Sequencing Kit (Applied Biosystems).

ClustalW program was used to determine editing sites, aligning DNA and cDNA sequences.

Sequence of cox3 gene were deposited in GeneBank database (accession number: FR669448).

\section{Results and Discussion}

\subsection{Analysis of Editing Sites on Cox3 Transcripts}

The Figure 2 shows the comparison of mitochondrial sequence of $\operatorname{cox} 3$ gene with the corresponding cDNA. Table 1 shows editing data about some cox3 mitochondrial genes of different plant species.

The analysis of results obtained in this investigation shows some specific features for the A. nidus mitochon-

Table 1. Comparison of editing value in cox3 mitochondrial transcripts between some land plants.

\begin{tabular}{|c|c|c|c|c|c|}
\hline & Editing C-U & J Editing U-C & Site Tot & $\%$ & Gene bp \\
\hline P. patens & 1 & & 1 & 0.1 & 798 \\
\hline B. vulgaris & 4 & & 4 & 0.5 & 798 \\
\hline B. napus & 7 & & 7 & 0.9 & 798 \\
\hline A. thaliana & 8 & & 8 & 1.0 & 798 \\
\hline T. aestivum & 12 & 1 & 13 & 1.6 & 798 \\
\hline O. sativa & 1 & & 1 & 0.1 & 843 \\
\hline A. nidus & 19 & 13 & 32 & 4.0 & 798 \\
\hline
\end{tabular}




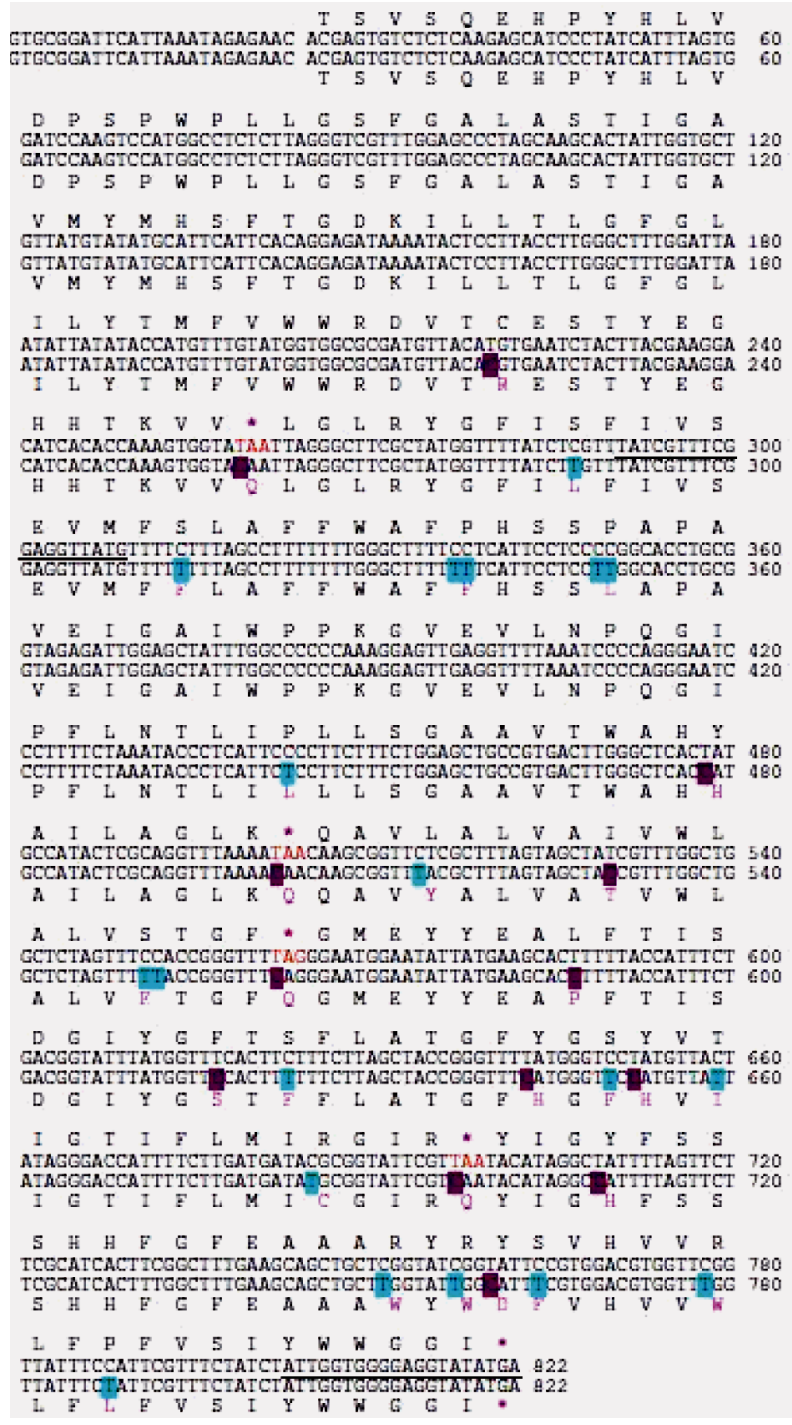

Figure 2. Alignment of genome sequence (upper) with cDNA counterpart of $\operatorname{cox} 3$ mitochondrial gene of $A$. nidus. Nucleotides underlined correspond to sequencing primers.

drial cox3 gene:

1) for the transcript of the gene, direct editing events (19 C-U; 2,3\%) and reverse editing events (13 U-C; 1,7\%) are almost equivalent;

2) the editing events have a high relevance: they suppress four stop codons within the cox3 gene;

3) 25 amino acids changes and 4 suppressions of stop codons (3 TAA-1 TAG) are found. These stop codons are corrected by $\mathrm{U}$ to $\mathrm{C}$ editing to sense codons of the amino acid Q (Figure 3);

4) in particularly critical positions, editing events of both types generate transcripts which can be translated as functional proteins;

5) the initial codon of $\operatorname{cox} 3$ is an ACG codon which is

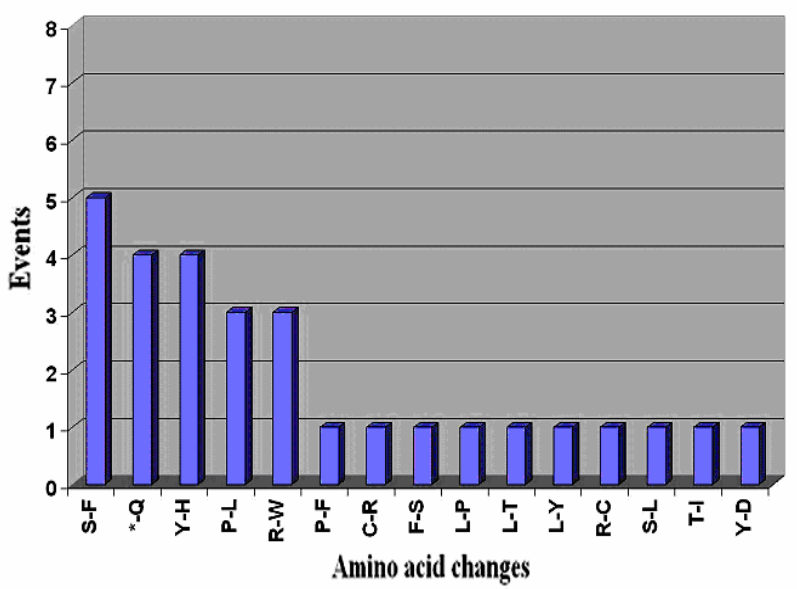

Figure 3. Amino acids changes after editing events in $A$. nidus cox3 mitochondrial transcript.

not changed to AUG by a direct editing event.

This observation can be considered in agreement with the finding of Dong F. G. [17] who found that the radish (Raphanus sativus L.) mitochondrial cox2 gene contains an ACG at the predicted translation initiation site which is not converted to AUG codon in the mRNA, although 15 other RNA editing sites were identified. So our finding confirms that in plant mitochondria ACG codon can be utilized as initiation codon;

6) cox3 editing events have a higher frequency on $A$. nidus transcripts compared with the same genes studied on other angiosperm genomes (Table 1);

7) reverse editing events (U-C) are considerably more frequent in cox3 transcripts of $A$. nidus than in other plant species, where they are nearly absent (Table 1);

8) as shown in Figure 4, for 17 codons changes occur in first position, for 13 codons in second position and for only one codon in third position.

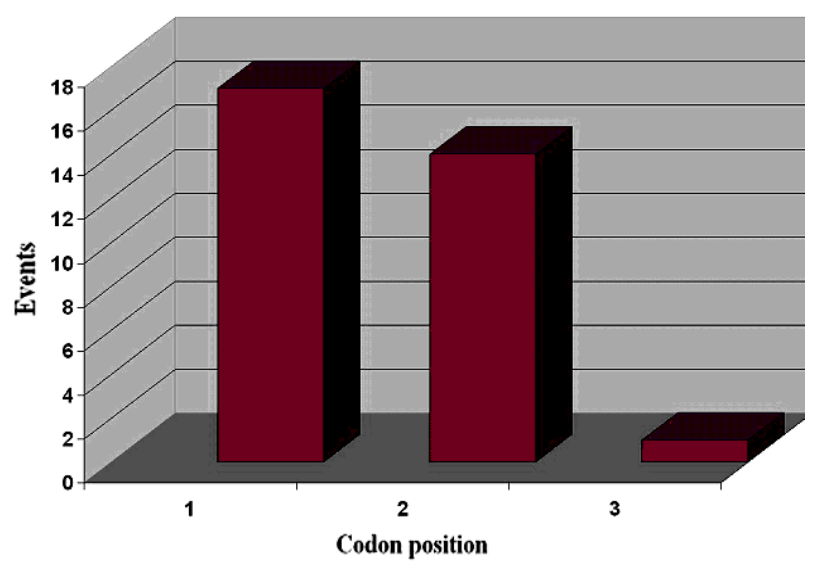

Figure 4. Distribution of editing events across codon positions. 


\section{REFERENCES}

[1] K. Oda, K. Yamato, E. Ohta, H. Fukuzawa, T. Kohchi, S. Nakayama, K. Ishizaki, M. Fujisawa and K. Yamato, "Gene Organization Deduced from the Complete Sequence of Liverwort Marchantia polymorpha Mitochondrial DNA. A Primitive Form of Plant Mitochondrial Genome,” Journal of Molecular Biology, Vol. 223, No. 1, 1992, pp. 1-7. doi:10.1016/0022-2836(92)90708-R

[2] K. Terasawa, M. Odahara, Y. Kabeya, T. Kikugawa, T. Sekine, M. Fujiwara and N. Sato, "The Mitochondrial Genome of the Moss Physcomitrella patens Sheds New Light on Mitochondrial Evolution in Land Plants," Molecular Biology and Evolution, Vol. 24, No. 3, 2006, pp. 699-709. doi:10.1093/molbev/msl198

[3] M. Unseld, J. R. Marienfeld, P. Brandt and A. Brennicke, "The Mitochondrial Genome of Arabidopsis thaliana Contains 57 Genes in 366,924 Nucleotides," Nature Genetics, Vol. 15, 1997, pp. 57-61. doi:10.1038/ng0197-57

[4] T. Kubo, S. Nishizawa, A. Sugawara, N. Itchoda, A. Estiati and T. Mikami, "The Complete Nucleotide Sequence of the Mitochondrial Genome of Sugar Beet (Beta vulgaris L.) Reveals a Novel Gene for tRNA(Cys) (GCA)," Nucleic Acids Research, Vol. 28, No. 13, 2000, pp. 2571-2576. doi:10.1093/nar/28.13.2571

[5] Y. Notsu, S. Masood, T. Nishikawa, N. Kubo, G. Akiduki, M. Nakazono, A. Hirai and K. Kadowaki, "The Complete Sequence of the Rice (Oryza sativa L.) Mitochondrial Genome: Frequent DNA Sequence Acquisition and Loss dur- ing the Evolution of Flowering Plants," Molecular Genetics and Genomics, Vol. 268, No. 4, 2002, pp. 434-445. doi:10.1007/s00438-002-0767-1

[6] H. Handa, "The Complete Nucleotide Sequence and RNA Editing Content of the Mitochondrial Genome of Rapeseed (Brassica napus L.): Comparative Analysis of the Mitochondrial Genomes of Rapeseed and Arabidopsis thaliana," Nucleic Acids Research, Vol. 31, No. 20, 2003, pp. 5907-5916. doi:10.1093/nar/gkg795

[7] S. W. Clifton, P. Minx, C. M. Fauron, M. Gibson, J. O. Allen, H. Sun, M. Thompson, W. B. Barbazuk, S. Kanuganti, C. Tayloe, L. Meyer, R. K. Wilson and K. J. Newton, "Sequence and Comparative Analysis of the Maize NB Mitochondrial Genome," Plant Physiology, Vol. 136, 2004, pp. 3486-3503. doi:10.1104/pp.104.044602

[8] Y. Sugiyama, Y. Watase, M. Nagase, N. Makita, S. Yagura, A. Hirai and M. Sugiura, "The Complete Nucleotide Sequence and Multipartite Organization of the Tobacco Mitochondrial Genome: Comparative Analysis of Mitochondrial Genomes in Higher Plants, Molecular Genetics and Genomics, Vol. 272, No. 6, 2005, pp. 603-615.

\section{$\underline{\text { doi: } 10.1007 / s 00438-004-1075-8}$}

[9] Y. Ogihara, Y. Yamazaki, K. Murai, A. Kanno, T. Terachi, T. Shiina, N. Miyashita, S. Nasuda, C. Nakamura, N. Mori, S. Takumi, M. Murata, S. Futo and K. Tsunewaki, "Structural Dynamics of Cereal Mitochondrial Genomes as Revealed by Complete Nucleotide Sequencing of the Wheat Mitochondrial Genome," Nucleic Acids Research, Vol. 33, No. 19, 2005, pp. 6235-6250. doi:10.1093/nar/gki925

[10] A. Kanno, M. Nakazono, A. Hirai and T. Kameya, “A Chloroplast Derived trnH Gene Is Expressed in the Mitochondrial Genome of Gramineous Plants,” Plant Molecular Biology, Vol. 34, No. 2, 1997, pp. 353-356. doi:10.1023/A:1005828728036

[11] J. Fey and L. Marechal-Drouard, "Expression of the Two Chloroplast-Like tRNA (Asn) Genes in Potato Mitochondria: Mapping of Transcription Initiation Sites Present in the trnN1-trnYnad2 Cluster and Upstream of trnN2," Current Genetics, Vol. 36, No. 1-2, 1999, pp. 49-54. doi:10.1007/s002940050471

[12] F. Damiano, L. R. Ceci, L. Siculella and R. Gallerani, “Transcription of Two Sunflower (Helianthus annuus L.) Mitochondrial tRNA Genes Having Different Genetic Origins,” Gene, Vol. 286, No. 1, 2002, pp. 25-32. doi:10.1016/S0378-1119(01)00801-0

[13] S. Panarese, G. Rainaldi, C. De Benedetto and R. Gallerani, "Sequencing of a Segment of a Monilophyte Species Mitochondrial Genome Reveals Features Highly Similar to those of Seed Plant mtDNAs,” The Open Plant Science Journal, Vol. 2, 2008, pp. 15-20. doi:10.2174/1874294700802010015

[14] M. R. Hanson, M. L. Boeshore, P. McClean, M. O’Connell and $\mathrm{H}$. Nivison, "The Isolation of Mitochondria and Mitochondrial DNA, Methods in Enzymology, Vol. 118, 1986, pp. 437-447. doi:10.1016/0076-6879(86)18091-8

[15] P. Taberlet, L. Gielly, G. Pautou and J. Bouvet, "Universal Primers for Amplification of the Non-Coding Regions of Chloroplast DNA,” Plant Molecular Biology, Vol. 17, No. 5, 1991, pp. 1105-1109. doi:10.1007/BF00037152

[16] K. Isono, Y. Niwa, K. Satoh and H. Kobayashi, "Evidence for Transcriptional Regulation of Plastid Photosynthesis Genes in Arabidopsis thaliana Roots," Plant Physiology, Vol. 114, No. 2, 1997, pp. 623-630. doi:10.1104/pp.114.2.623

[17] F. G. Dong, K. G. Wilson and C. A. Makaroff, "The Radish (Raphanus sativus L.) Mitochondrial cox2 Gene Contains an ACG at the Predicted Translation Initiation Site,” Current Genetics, Vol. 34, No. 2, 1998, pp. 79-87. doi:10.1007/s002940050369 